\title{
Correlation of Treadmill Stress Test with Coronary Angiography to Predict Coronary Artery Disease in Males versus Females
}

\author{
B. L. Maneesh Kumar ${ }^{1}$ Edavaluru Bhuvaneshwari ${ }^{1}$ \\ ${ }^{1}$ Department of Pharmacology, Osmania Medical College, Koti, \\ Hyderabad, Telangana, India \\ Indian J Cardiovasc Dis Women-WINCARS 2017;2:25-28.
}

\begin{abstract}
Address for correspondence B. L. Maneesh Kumar, Department of Pharmacology, Osmania Medical College, Koti, Hyderabad 500095, Telangana, India (e-mail: Pinctada64@gmail.com).
\end{abstract}

\begin{abstract}
Background Treadmill stress test (TMT) is the most commonly performed stress test for diagnosis of coronary artery disease (CAD) in outpatient setting. The present study was conducted to find correlation of TMT with coronary angiography (CAG) in males versus female patients.

Methods Total 422 patients who had undergone CAG and TMT were enrolled in present study. TMT test was done using Bruce protocol, and results were classified as inconclusive, low, intermediate, and high probability for inducible ischemia.

Results Of 422 patients enrolled in the present study, 290 (69\%) were males and 132 (31\%) were females. Clinical presentation was angina on exertion CCS class II-III in 302 (71.6\%) patients and angina equivalent in 124 (29.4\%) patients. Out of these, 211 (50.2\%) were diabetic and 308 (72.9\%) were hypertensive. TMT was inconclusive, low, intermediate, and high probability for inducible ischemia in 35 (8.2\%), 40 (9.7\%), 19 (4.5\%), and $328(77.7 \%)$ patients, respectively. CAGs were normal in 149 (35.4\%) patients and abnormal in $273(64.6 \%)$ patients. Out of 328 patients with strongly positive TMT, 104 (31.7\%) had normal coronaries, 56 (17\%) had mild CAD, while 75 (22.8\%) had SVD. LMCA disease, DVD, and TVD were found in $5(1.5 \%), 48(14.6 \%)$, and $40(12.1 \%)$ patients, respectively. Of 132 female patients, $70(53 \%)$ were found to have CAD and 62 (47\%) had normal coronaries on CAG. Of 290 males, 241(83.1\%) were found to have CAD and 49 (16.8\%) had normal coronaries on CAG. Positive predictive

Keywords

- coronary artery disease

- coronary angiography

- treadmill stress test value (PPV) in females was $53 \%$ and in males was $83 \%$. Subgroup analysis showed that if there was high-probability TMT along with older age $(p<0.00)$, male sex $(p<0.01)$, and diabetes $(p<0.00)$, chances of having CAD were more.

Conclusion PPV of positive TMT in female is $53 \%$ and in male is $83 \%$. High-probability TMT along with the older age, male sex, and presence of diabetes mellitus (DM) makes the probability of having $C A D$ is high.
\end{abstract}

\section{Introduction}

In the emerging health care scenario in developing countries, urbanized lifestyle changes are resulting in increased prevalence of cardiovascular risk factors (diabetes, obesity, hypertension [HTN], and hypercholesterolemia). These in turn result in cardiovascular diseases (CVDs) that include coronary heart disease and stroke. ${ }^{1}$

The accuracy of the treadmill stress test (TMT) in predicting significant coronary heart disease is variable, depending
DOI https://doi.org/ 10.1055/s-0037-1607032.
Copyright (c) 2017 Women in Cardiology and Related Sciences
License terms

(๑) $\Theta \circledast$ 
in part on the "pretest likelihood" of coronary heart disease (also known as Bayes' theorem). In a person at high risk for coronary heart disease (e.g., advanced age, multiple coronary risk factors), an abnormal TMT is very predictive of the presence of coronary heart disease (>90\% accurate). ${ }^{2}$ In the present study with changing risk factor profiles along with increasing incidence of CAD in younger population (even as young as 20 years), we want to see the predictability of CAD in females versus males by TMT.

\section{Study Design and Methodology}

This was a prospective study done in our Institution from 2014 to 2015, after obtaining ethical clearance from our institutional ethical committee, and informed consent was got from all patients. In this study, we recruited the male and female patients who underwent coronary angiogram (CAG) along with positive TMT.

The risk factors taken into consideration for the study included weight, presence/absence of HTN, presence/absence of diabetes mellitus (DM), smoking, and family history of CAD. A patient was considered as diabetic if the fasting blood glucose was $\geq 126 \mathrm{mg} / \mathrm{dL}$ or if the 2-hour (postprandial) blood glucose level was $\geq 200 \mathrm{mg} / \mathrm{dL}$, or if the hemoglobin $\mathrm{A}_{1 \mathrm{c}}\left(\mathrm{HbA}_{1 \mathrm{c}}\right)$ (glycosylated $\mathrm{Hb}$ ) was $\geq 6.5 \%$. Family history of premature CAD in first-degree relatives was defined as ischemic heart disease (IHD) or sudden cardiac death before 55 years of age (in men) or 65 years of age (in women). A patient was considered as hypertensive if the systolic blood pressure was $\geq 140 \mathrm{~mm} \mathrm{Hg}$ or the diastolic blood pressure was $\geq 90 \mathrm{~mm} \mathrm{Hg}$, according to the Joint National Committee (JNC) VII guidelines. ${ }^{3}$ Current smoking status was noted. TMT was done using Bruce protocol, and results were evaluated as inconclusive, low, intermediate, high probability for inducible ischemia. ${ }^{4}$ Details of the CAG were noted. CAG was classifies as normal or abnormal. Abnormal CAG includes mild CAD, single-vessel disease (SVD), double-vessel disease (DVD), triple-vessel disease (TVD), and left main coronary artery (LMCA) disease.

\section{Statistical Analysis}

Data analysis was performed using Minitab version 17 software (Pennsylvania State University, Philadelphia, United States). Categorical variables were compared using chi-square test. Continuous variables were expressed as mean \pm standard deviation (SD) and were compared by the Student's $t$-test. Nominal logistic regression analysis was performed to determine the significant parameters (e.g., age, DM, HTN, smoking [SM]) that influence the increase in positivity of CAG. Results with a $p$ value $<0.05$ are considered to be significant.

\section{Results}

Of 422 patients enrolled in present study, 290 (69\%) were males and 132 (31\%) were females (-Fig.1). Comparison of the demographic features between the males and females is mentioned in - Table 1. Male patients were older, had more

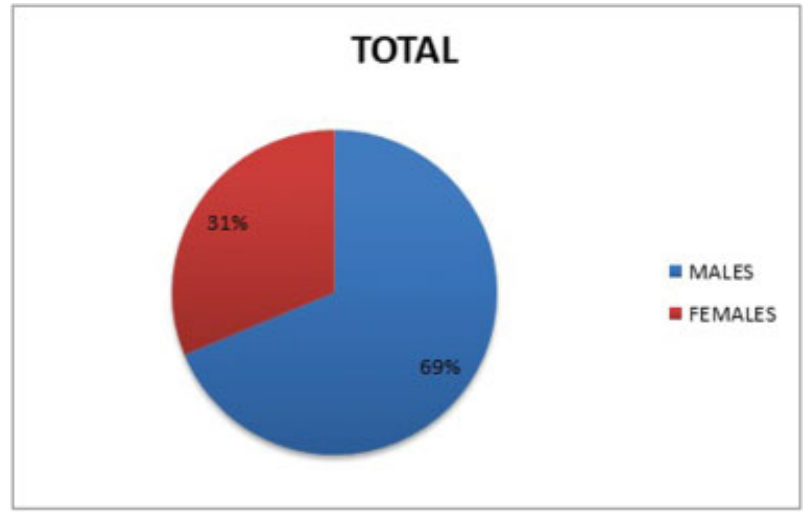

Fig. 1 Pie diagram depicting sex distribution of study population.

frequent HTN, and were smokers. However, there was no difference in mean systole and diastole blood pressure among male and female patients. Incidence of DM was comparable between both sexes. On 2D echocardiogram, regional wallmotion abnormalities (RWMAs) were more in males $(p=0.004)$ without difference in ejection fraction (EF).

Clinical presentation and indication to do CAG were exertion angina class II-III in 302 (71.6\%) patients and angina equivalent (dyspnea) in 124 (29.4\%) patients. The results of TMT were inconclusive, low, intermediate, and high probability for inducible ischemia in 35 (8.2\%), 40 (9.7\%), 19 (4.5\%), and 328 (77.7\%) patients, respectively (-Fig. 2).

CAGs were normal in 149 (35.4\%) patients and abnormal in 273 (64.6\%) patients. Mild CAD, SVD, DVD, TVD, and LMCA disease (-Fig. 3) were found in 74 (27.1\%), 90 (32.9\%), 59 (21.6\%) 45 (16.4\%), and 5 (1.8\%) patients, respectively.

Out of 328 patients with strongly positive TMT (-Fig. 4), 104 (31.7\%) had normal coronaries, 56 (17\%) had mild CAD, whereas 75 (22.8\%) had SVD. LMCA disease, DVD, and TVD were found in 5 (1.5\%), 48 (14.6\%), and 40 (12.1\%) patients, respectively.

Of 132 female patients, 70 (53\%) were found to have abnormal CAG and 62 (47\%) were normal. Of 290 males,

Table 1 Demographic and 2D echocardiographic features of study population

\begin{tabular}{|l|l|l|l|}
\hline Variable & Female & Male & $p$ Value \\
\hline Age (y) & $52.1 \pm 8.9$ & $57.1 \pm 10.6$ & 0.000 \\
\hline $\begin{array}{l}\text { Systolic pressure } \\
\text { (mm Hg) }\end{array}$ & $157.9 \pm 28.8$ & $159.9 \pm 74.2$ & 0.700 \\
\hline $\begin{array}{l}\text { Diastolic pressure } \\
\text { (mm Hg) }\end{array}$ & $78.6 \pm 10.9$ & $77.5 \pm 11.2$ & 0.331 \\
\hline HTN (\%) & $88(66.6 \%)$ & $284(97.9 \%)$ & 0.000 \\
\hline DM (\%) & $60(45.5 \%)$ & $151(52.1 \%)$ & 0.206 \\
\hline Smoker (\%) & $2(1.5 \%)$ & $54(18.6 \%)$ & 0.000 \\
\hline RWMA & $7(5.3 \%)$ & $88(13.4 \%)$ & 0.004 \\
\hline LV dysfunction & $5(3.7 \%)$ & $23(7.9 \%)$ & 0.071 \\
\hline
\end{tabular}

Abbreviations: DM, diabetes mellitus; HTN, hypertension; LV, left ventricular; RWMA, regional wall-motion abnormality. 


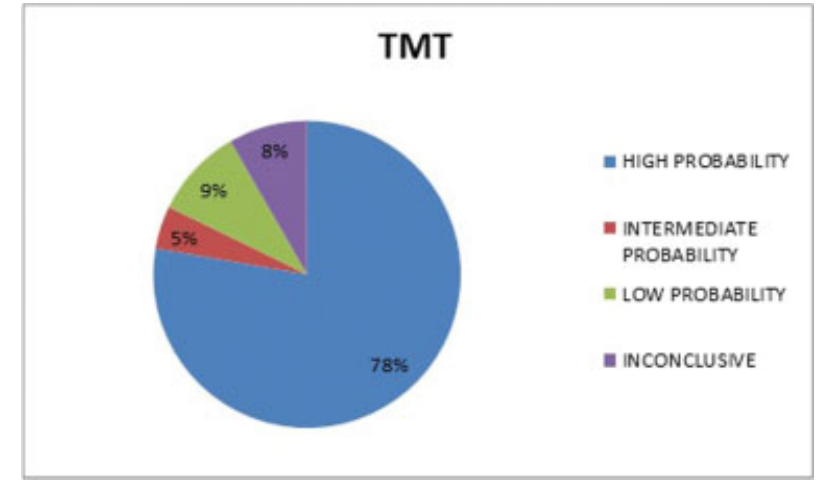

Fig. 2 Pie diagram depicting probability of inducible ischemia on TMT in study population. TMT, treadmill stress test.

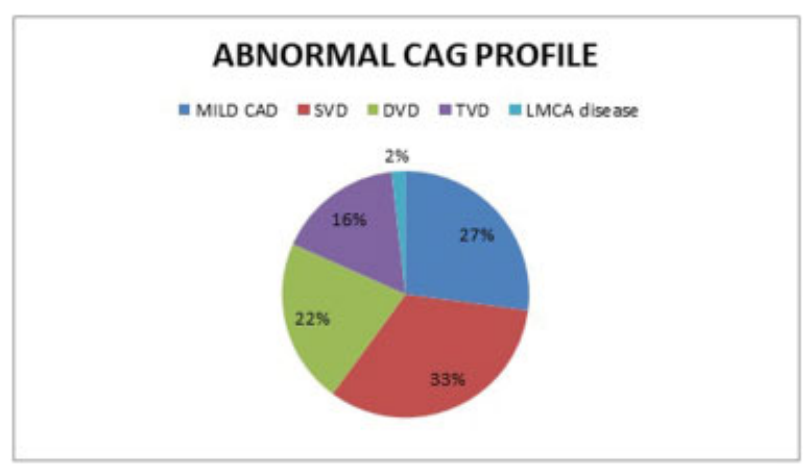

Fig. 3 Pie diagram depicting abnormal CAG profile in study population. CAD, coronary artery disease; CAG, coronary angiography; DVD, doublevessel disease; LMCA, left main coronary artery; SVD, single-vessel disease; TVD, triple-vessel disease.

241 (83.1\%) were found to have abnormal CAG and 49 (16.8\%) were normal ( $\mathbf{F i g . 5 A , ~ B ) . ~}$

The positive predictive value (PPV) of TMT for detection of CAD on CAG was $53 \%$ in females and $83 \%$ in males (- Fig. 6).

Nominal logistic regression was done to determine the factors that increase the probability of detection of CAD in high probable TMT patient. If there was high-probability TMT with older age $(p<0.00)$, male sex $(p<0.01)$, and diabetes $(p<0.00)$, chances of having CAD were more (-Table 2$)$.

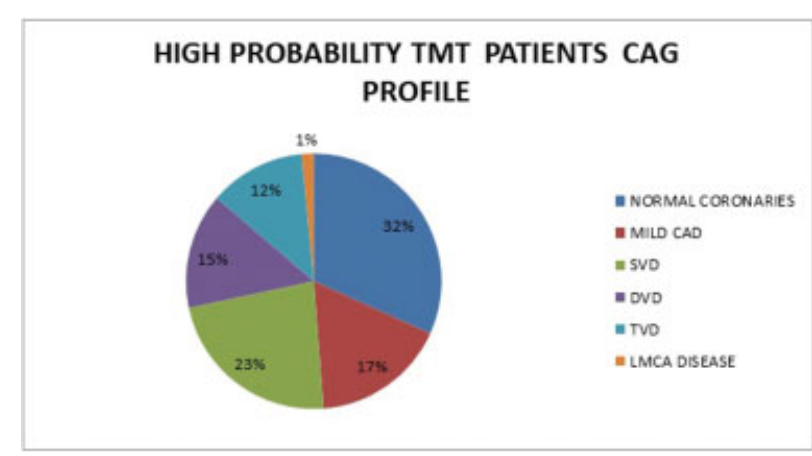

Fig. 4 Distribution of abnormal CAG profiles in high-probability treadmill stress test (TMT) patients. CAD, coronary artery disease; CAG, coronary angiography; DVD, double-vessel disease; LMCA, left main coronary artery; SVD, single-vessel disease; TMT, treadmill stress test; TVD, triple-vessel disease.

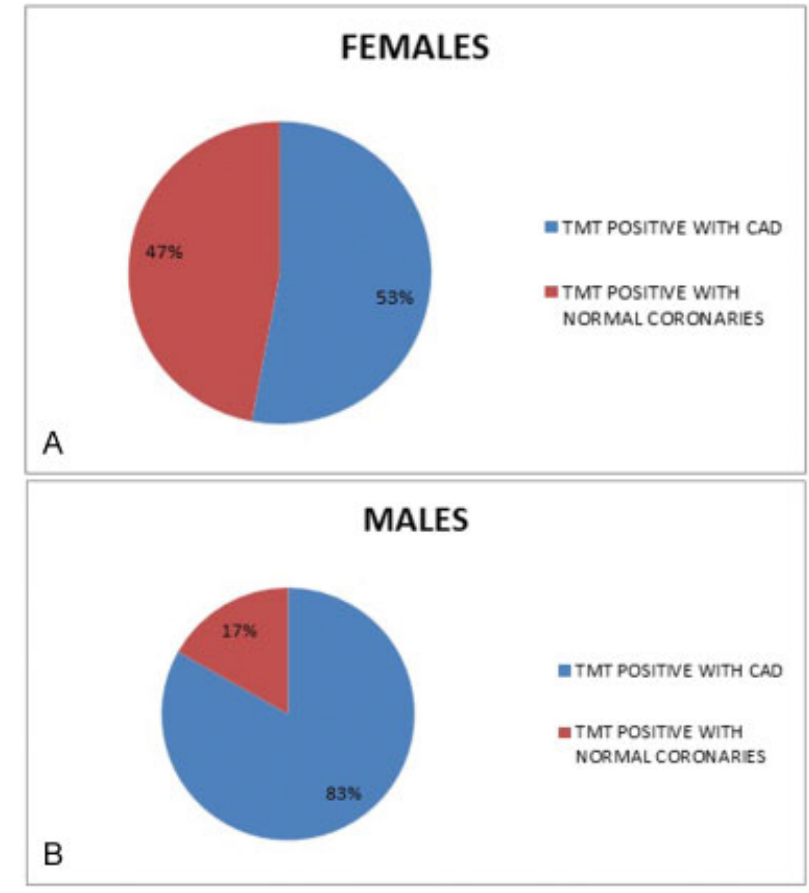

Fig. 5 (A) Pie diagram depicting CAG profile in TMT-positive females. (B) Pie diagram depicting CAG profile in TMT-positive males. CAD, coronary artery disease; CAG, coronary angiography; TMT, treadmill stress test.

\section{Discussion}

Accurate screening and diagnosis of IHD is one of the most important mechanisms of timely intervention and prevention of mortality. TMT and/or myocardial perfusion imaging (MPI) scan are used in many centers for screening to be followed up with CAG, which is used as a gold standard for the diagnosis of CAD. Now with the availability of improved equipment and radiotracers, the present-day role of these noninvasive investigations needs to be restudied.

TMT is the most widely used cardiac stress test. This is described as poor man's angiogram. In a person at high risk for coronary heart disease (e.g., advanced age, multiple coronary risk factors), an abnormal TMT is highly predictive of the presence of coronary heart disease ( $>90 \%$ accurate).

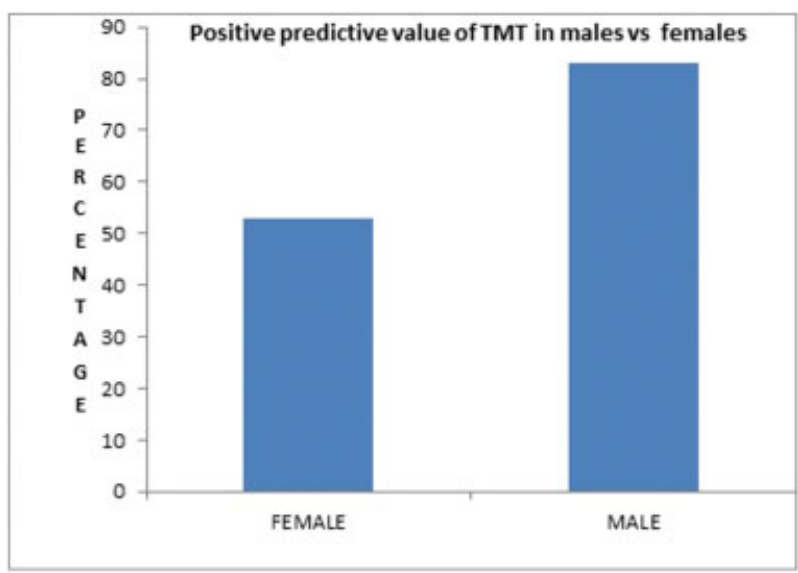

Fig. 6 Bar diagram depicting PPV in males and females. PPV, positive predictive value; TMT, probability treadmill stress test. 
Table 2 Nominal logistic regression analysis

\begin{tabular}{|l|l|l|l|l|}
\hline Source & Adj. dev & Adj. mean & Chi-square & $p$ Value \\
\hline Regression & 48.4 & 5.37 & 48.36 & 0.000 \\
\hline Age (y) & 4.05 & 4.05 & 4.05 & 0.044 \\
\hline Systolic pressure $(\mathrm{mm} \mathrm{Hg})$ & 1.94 & 1.89 & 1.89 & 0.169 \\
\hline Diastolic pressure $(\mathrm{mm} \mathrm{Hg})$ & 0.50 & 0.50 & 0.50 & 0.478 \\
\hline Sex & 6.67 & 6.67 & 6.67 & 0.010 \\
\hline HTN & 2.88 & 2.88 & 2.88 & 0.090 \\
\hline DM & 13.16 & 13.15 & 13.16 & 0.000 \\
\hline Smoker & 0.016 & 0.016 & 0.02 & 0.898 \\
\hline RWMA & 1.58 & 1.58 & 1.58 & 0.208 \\
\hline LV dysfunction & 1.43 & 1.43 & 1.43 & 0.232 \\
\hline
\end{tabular}

Abbreviations: DM, diabetes mellitus; HTN, hypertension; LV, left ventricular.

However, a relatively normal TMT may not reflect the absence of significant disease in a person with the same risk factors. Conversely, in a person with a low-risk, a normal TMT is very predictive of the absence of significant coronary heart disease ( $>90 \%$ accurate), but an abnormal test may not reflect the true presence of coronary heart disease (so-called "false-positive TMT").

Even though the TMT remains a cost-effective, easily available, and widely applicable approach for early diagnosis of CAD, it has a relatively low sensitivity and specificity. Identification of CAD in women can be a diagnostic challenge because of several factors, including the lower prevalence of obstructive CAD in women younger than 65 years, more atypical manifestations of ischemic symptoms, and more frequent resting ST changes. False-positive TMT are common in female patients. The most common factors include anemia, ST changes, and $\mathrm{T}$-wave inversion in leads $\mathrm{V}_{2}-\mathrm{V}_{4}$ in $50 \%$ of female patients mostly related to anemia. Therefore, we wanted to study in our female population. According to Khanam et al from indoor, out of 50 TMT-positive female patients, 12 were having CAD on angiography (24\%) indicating a low predictive value of TMT. The prevalence of CAD was 70\% in high pretest probability group compared with $5 \%$ in low pretest probability group indicating high predictive value of TMT in high pretest probability group. SVD was most common (58\%) followed by TVD (25\%) and DVD (17\%). ${ }^{5}$ Similar findings ere revealed by Ismai. ${ }^{6}$ In our series $53 \%$ of females had normal coronaries even with positive TMT.

Kim et al, in their study, suggested that in the interest of cost-effectiveness, screening for asymptomatic CAD could be limited to elderly patients with a duration of diabetes $\geq 10$ years. ${ }^{7}$ In the present study, only high probability of TMT positivity is not sufficient to predict the obstructive coronary artery disease (CAD) on CAG. Along with TMT positivity, if older age and diabetes also are present, the likelihood of CAD detection increases.

Periyapattana and AVS from south India and Stanley suggested in their study that combined TMT and MPI in- creases the PPV to $94 \%$ and diagnostic accuracy to $88 \%$ and together performs the gatekeeper function for CAG so that optimal decision making can be achieved., ${ }^{8,9}$

Our study showed that PPV of TMT is lower (53\%) in females than in males (83\%). To detect obstructive CAD by CAG, better to see the other parameters also such as age and diabetes along with high-probability TMT.

\section{Acknowledgment}

Dr. M. Jyotsna, Professor and HOU-IV, Department of Cardiology, Nizam's Institute of Medical Sciences, Punjagutta, Telangana.

\section{References}

1 Sanchis-Gomar F, Perez-Quilis C, Leischik R, Lucia A. Epidemiology of coronary heart disease and acute coronary syndrome. Ann Transl Med 2016;4(13):256

2 Ben-Shlomo Y, Brookes S, Hickman M. Epidemiology, EvidenceBased Medicine and Public Health. 6th ed. Oxford, UK: WileyBlackwell; 2013

3 JNC VII guidelines for hypertension. Hypertension 2003;42:1206

4 Nahormek PA, Chahine RA, Raizner AE, et al. The magnitude of exercise-induced ST segment depression and the predictive value of exercise testing. Clin Cardiol 1979;2(04):286-290

5 Khanam B, Bansal R, Bansal A. An evaluation of coronary artery disease by coronary angiography in TMT positive female patients. J Evid Based Med Health Care 2017;4(25):1460-1462

6 Ismai M, Andrab SM, Rashi A, et al. Coronary artery profile on coronary angiography in TMT positive female patients. J Evol Med Dental Sci 2015;4:100

$7 \mathrm{Kim} \mathrm{MK}$, Baek KH, Song $\mathrm{KH}$, et al. Exercise treadmill test in detecting asymptomatic coronary artery disease in type 2 diabetes mellitus. Diabetes Metab J 2011;35(01):34-40

8 Periyapattana GK, AVS AK. Correlation of TMT, MPI \& Coronary angiography - our experience at a tertiary care hospital. Paper presented at: International Conference on Integrated Medical Imaging in Cardiovascular Diseases; October 10 14, 2016; Vienna. Available at https://conferences.iaea.org/indico/event/100/contribution/34

9 George S. Angiographic profile and treadmill test relationship of women with chest pain suggestive of coronary artery disease. World J Cardiovasc Dis 2017;7(08):225-232 